\title{
Coronavirus disease 2019 (COVID-19) research agenda for healthcare epidemiology
}

Lona Mody MD, MSc ${ }^{1,2}$, Ibukunoluwa C. Akinboyo $\mathrm{MD}^{3}$, Hilary M. Babcock MD, MPH, FIDSA FSHEA ${ }^{4}$,

Werner E. Bischoff MD, MS, PhD, FSHEA ${ }^{5}$, Vincent Chi-Chung Cheng MD $^{6,7}$, Kathleen Chiotos MD, MSCE ${ }^{8}$, Kimberly C. Claeys PharmD ${ }^{9}$, K. C. Coffey MD, MPH ${ }^{10}$, Daniel J. Diekema MD, MS ${ }^{11}$, Curtis J. Donskey MD ${ }^{12,13}$, Katherine D. Ellingson $\mathrm{PhD}^{14}$, Heather M. Gilmartin PhD, NP, CIC, FAPIC ${ }^{15,16}$, Shruti K. Gohil MD, MPH ${ }^{17,18}$, Anthony D. Harris MD, MPH, FIDSA, FSHEA ${ }^{10}$, Sara C. Keller MD, MPH, MSHP ${ }^{19}$, Eili Y. Klein MA, PhD ${ }^{20}$, Sarah L. Krein PhD, RN ${ }^{21,22}$, Jennie H Kwon DO, $\mathrm{MSCl}^{4}$, Adam S. Lauring MD, $\mathrm{PhD}^{23}$, Daniel J. Livorsi MD ${ }^{24,11}$, Eric T. Lofgren MSPH, PhD ${ }^{25}$, Katreena Merrill PhD, RN ${ }^{26}$, Aaron M. Milstone MD, $\mathrm{MHS}^{27}$, Elizabeth A. Monsees PhD, MBA, RN, CIC, FAPIC ${ }^{28,29}$, Daniel J. Morgan MD, MS ${ }^{10,30}$, Luci P. Perri RN, MSN, MPH, CIC, FAPIC, CSPDT ${ }^{31}$, Christopher D. Pfeiffer MD, MHS ${ }^{32,33}$, Clare Rock MD, MS ${ }^{19}$, Sanjay Saint MD, MPH ${ }^{34,22}$, Emily Sickbert-Bennett PhD, MS, CIC, FSHEA ${ }^{35}$, Felicia Skelton MD, MS ${ }^{36,37}$, Katie J. Suda PharmD, MS ${ }^{38,39}$, Thomas R. Talbot MD, $\mathrm{MPH}^{40}$, Valerie M. Vaughn MD, $\mathrm{MSc}^{41}$, David J. Weber MD, MPH, FIDSA, FSHEA ${ }^{42}$, Timothy L. Wiemken PhD, MPH, FAPIC, FSHEA, $\mathrm{CIC}^{43}$, Mohamed H. Yassin MD, PhD ${ }^{44,45}$, Matthew J. Ziegler MD, MSCE ${ }^{46}$, and Deverick J. Anderson MD, MPH ${ }^{47}$ on behalf of the SHEA Research Committee

${ }^{1}$ Division of Geriatric and Palliative Medicine, Department of Internal Medicine, University of Michigan, Ann Arbor, Michigan, United States, ${ }^{2}$ Geriatrics Research Education and Clinical Center, Veterans' Affairs Ann Arbor Healthcare System, Ann Arbor, Michigan, United States, ${ }^{3}$ Division of Infectious Diseases, Department of Pediatrics, Duke University School of Medicine, Durham, North Carolina, United States, ${ }^{4}$ Washington University School of Medicine, St. Louis, Missouri, United States, ${ }^{5}$ Wake Forest School of Medicine, Winston Salem, North Carolina, United States, ${ }^{6}$ Department of Microbiology, Queen Mary Hospital, Hong Kong Special Administrative Region, China, ${ }^{7}$ Infection Control Team, Queen Mary Hospital, Hong Kong West Cluster, Hong Kong Special Administrative Region, China, ${ }^{8}$ Division of Critical Care Medicine, Children's Hospital of Philadelphia, Philadelphia, Pennsylvania, United States, ${ }^{9}$ University of Maryland School of Pharmacy, Baltimore, Maryland, United States, ${ }^{10}$ University of Maryland School of Medicine, Baltimore, Maryland, United States, ${ }^{11}$ Carver College of Medicine, University of lowa, lowa City, lowa, United States, ${ }^{12}$ Infectious Diseases Section, Louis Stokes Cleveland Veterans' Affairs Medical Center, Cleveland, Ohio, United States, ${ }^{13}$ Case Western Reserve University School of Medicine, Cleveland, Ohio, United States, ${ }^{14}$ Department of Epidemiology and Biostatistics, College of Public Health, University of Arizona, Tucson, Arizona, United States, ${ }^{15}$ Veterans' Affairs Eastern Colorado Healthcare System, Aurora, Colorado, United States, ${ }^{16}$ Colorado School of Public Health, University of Colorado, Aurora, Colorado, United States, ${ }^{17}$ Division of Infectious Diseases, University of California Irvine School of Medicine, Irvine, California, United States, ${ }^{18}$ Epidemiology and Infection Prevention, UC Irvine Health, Irvine, California, United States, ${ }^{19}$ Division of Infectious Diseases, John Hopkins University School of Medicine, Baltimore, Maryland, United States, ${ }^{20}$ Department of Emergency Medicine, Johns Hopkins University, Baltimore, Maryland, Unites States, ${ }^{21}$ Veterans' Affairs Ann Arbor Center for Clinical Management Research, Ann Arbor, Michigan, United States, ${ }^{22}$ Department of Internal Medicine, University of Michigan, Ann Arbor, Michigan, United States, ${ }^{23}$ Division of Infectious Diseases, Department of Internal Medicine, University of Michigan, Ann Arbor, Michigan, United States, ${ }^{24}$ Iowa City Veterans' Affairs Health Care System, lowa City, lowa, United States, ${ }^{25}$ Paul G. Allen School for Global Animal Health, Washington State University, Pullman, Washington, United States, ${ }^{26}$ Brigham Young University, Provo, Utah, United States, ${ }^{27}$ Division of Pediatric Infectious Diseases, Johns Hopkins University School of Medicine, Baltimore, Maryland, United States, ${ }^{28}$ Children's Mercy Kansas City, Kansas City, Missouri, United States, ${ }^{29}$ University of Missouri-Kansas City School of Medicine, Kansas City, Missouri, United States, ${ }^{30}$ Veterans' Affairs Maryland Healthcare System, Baltimore, Maryland, United States, ${ }^{31}$ Infection Control Results, Wingate, North Carolina, United States, ${ }^{32}$ Veterans' Affairs Portland Health Care System, Portland, Oregon, United States, ${ }^{33}$ Oregon Health \& Science University, Portland, Oregon, United States, ${ }^{34}$ Veterans' Affairs Ann Arbor Healthcare System, Ann Arbor, Michigan, United States, ${ }^{35}$ Department of Infection Prevention, University of North Carolina Medical Center, Chapel Hill, North Carolina, United States, ${ }^{36}$ Michael E. DeBakey Veterans' Affairs Medical Center, Houston, Texas, United States, ${ }^{37} \mathrm{H}$. Ben Taub Department of Physical Medicine \& Rehabilitation, Baylor College of Medicine, Houston, Texas, United States, ${ }^{38}$ Center for Health Equity Research and Promotion, Veterans' Affairs Pittsburgh Healthcare System, Pittsburgh, Pennsylvania, United States, ${ }^{39}$ Division of General Internal Medicine, University of Pittsburgh School of Medicine, Pittsburgh, Pennsylvania, United States, ${ }^{40}$ Vanderbilt University School of Medicine, Nashville, Tennessee, United States, ${ }^{41}$ Division of General Internal Medicine, Department of Internal Medicine, University of Utah School of Medicine, Salt Lake City, Utah, United States, ${ }^{42}$ University of North Carolina at Chapel Hill, Chapel Hill, North Carolina, United States, ${ }^{43}$ Division of Infectious Diseases, Allergy,

Author for correspondence: Lona Mody, E-mail: lonamody@umich.edu. Or Deverick J. Anderson, E-mail: deverick.anderson@duke.edu

Cite this article: Mody L, et al. (2022). Coronavirus disease 2019 (COVID-19) research agenda for healthcare epidemiology. Infection Control \& Hospital Epidemiology, 43: 156-166, https://doi.org/10.1017/ice.2021.25

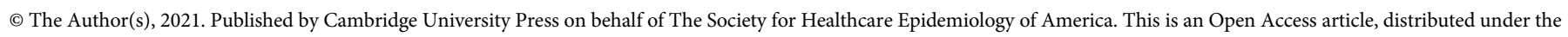

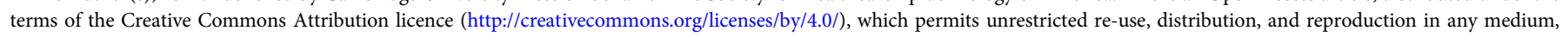
provided the original work is properly cited. 
and Immunology, Department of Medicine, Saint Louis University School of Medicine, St Louis, Missouri, United States, ${ }^{44}$ School of Medicine, University of Pittsburgh, Pittsburgh, Pennsylvania, United States, ${ }^{45}$ Infectious Diseases and Microbiology, Graduate School of Public Health, University of Pittsburgh, University of Pittsburgh, Pittsburgh, Pennsylvania, United States, ${ }^{46}$ Infectious Diseases Division, University of Pennsylvania, Philadelphia, Pennsylvania and ${ }^{47}$ Duke Center for Antimicrobial Stewardship and Infection Prevention, Duke University School of Medicine, Durham, North Carolina, United States

\section{Abstract}

This SHEA white paper identifies knowledge gaps and challenges in healthcare epidemiology research related to coronavirus disease 2019 (COVID-19) with a focus on core principles of healthcare epidemiology. These gaps, revealed during the worst phases of the COVID-19 pandemic, are described in 10 sections: epidemiology, outbreak investigation, surveillance, isolation precaution practices, personal protective equipment (PPE), environmental contamination and disinfection, drug and supply shortages, antimicrobial stewardship, healthcare personnel (HCP) occupational safety, and return to work policies. Each section highlights three critical healthcare epidemiology research questions with detailed description provided in supplementary materials. This research agenda calls for translational studies from laboratory-based basic science research to well-designed, large-scale studies and health outcomes research. Research gaps and challenges related to nursing homes and social disparities are included. Collaborations across various disciplines, expertise and across diverse geographic locations will be critical.

(Received 7 January 2021; accepted 7 January 2021; electronically published 25 January 2021)

The emergence and rapid worldwide spread of severe acute respiratory syndrome coronavirus 2 (SARS-CoV-2) has led to substantial social and economic disruption and loss of life. Throughout the pandemic, healthcare providers, hospitals, and health systems have worked tirelessly to provide safe care for patients while simultaneously ensuring safety for frontline providers. Early efforts to prevent transmission relied on prepandemic evidence and rapidly emerging novel data. Through the first 9 months of the pandemic, $>60,000$ articles were published on SARS-CoV-2 and coronavirus disease 2019 (COVID-19), not including the now ubiquitous preprints. ${ }^{1}$ As a result, the scientific community already has learned a great deal about COVID-19, leading to evolving guidelines for treatment, testing, and prevention. ${ }^{2-5}$ Despite considerable progress, the community still has much to learn.

As the adage goes, "The more you know, the more you realize how much you don't know." This SHEA white paper identifies remaining knowledge gaps and challenges in healthcare epidemiology research related to COVID-19. These gaps are described in 10 sections : epidemiology, outbreak investigation, surveillance, isolation precaution practices, personal protective equipment (PPE), environmental contamination and disinfection, drug and supply shortages, antimicrobial stewardship, healthcare personnel (HCP) occupational safety, and return to work policies (Table 1; Supplementary Tables 1-4 online). Each section highlights 3 critical healthcare epidemiology research questions, while many other important research questions are provided in supplementary tables, including a table highlighting the subset of questions particularly relevant to children (Supplementary Table 5 online). Research gaps and challenges related to nursing homes and social disparities are included.

\section{Epidemiology}

Understanding the epidemiology of SARS-CoV-2 is critical to minimizing the burden of the COVID-19 pandemic in healthcare settings. Epidemiologic research on individual as well as populationlevel transmission dynamics, risk factors for virus acquisition, and predictors of severe disease outcomes can inform healthcare capacity planning, clinical care, and infection prevention practices within healthcare settings. Three research domains identified below (Supplementary Table 1 online) represent priority areas with unanswered questions in the epidemiology of disease relevant to healthcare and infection prevention:
Priority area 1: Understand heterogeneity in epidemiology and transmission dynamics of SARS-CoV-2.

Priority area 2: Define characteristics and impact of asymptomatic/ pre-symptomatic patients infected with SARS-CoV-2.

Priority area 3: Characterize risk factors that lead to severe disease outcomes including age, sex, and race, with special emphasis on health disparities, socio-economic status, and comorbidities.

The heterogeneity in COVID-19 transmission dynamics is typified by both strain differences and "superspreading events" in which a small number of individuals account for a large fraction of transmission. Recent reports of variant strains being associated with an increased facility for spread as well as higher viral burdens in infected individuals require further explanation. Identifying the causes of superspreading in healthcare settings is key, especially in various settings that house high-risk populations such as in nursing homes and other long-term care facilities. ${ }^{6-8}$ Also critical is an enhanced understanding of viral transmission patterns through the air (in more depth than simply droplet versus airborne transmission) that can support evidence-based PPE, physical distancing, and ventilation policies, which currently vary across healthcare settings. ${ }^{6} \mathrm{~W}$ ith continued shortages in PPE, identifying the relative risk of occupational versus community exposures in $\mathrm{HCP}$ is essential to identify failures in occupational safety and implement comprehensive interventions to safeguard HCP.

One unique epidemiologic feature of COVID-19 is the sheer number of asymptomatic or presymptomatic cases reported, which has ranged from $1 \%$ to $>50 \%$ and has resulted in widespread increases in SARS-CoV-2 testing. ${ }^{6,9-15}$ Several studies have documented high viral loads in asymptomatic individuals, which suggests that they could be significant contributors to transmission and that symptom screening alone cannot contain transmission. ${ }^{9,16}$ This issue highlights the need for studies in children, who have lower rates of illness and hospitalization and gather in school and daycare settings (Supplementary Table 5 online). ${ }^{17}$ An understanding of the role of asymptomatic or presymptomatic individuals in transmission will influence societal considerations regarding opening schools, resuming economic activities such as opening gyms, and allowing social events such as having small and large gatherings.

Finally, studying the epidemiology of severe and post-acute disease can inform patient and HCP safety protocols, clinical practice guidelines, vaccine recommendations, and concurrent management of other chronic conditions. To date, the burden of 
Table 1. COVID-19 Healthcare Epidemiology Research Priorities

\begin{tabular}{|c|c|c|}
\hline Section & Research Priority Areas & Detailed Research Questions \\
\hline \multicolumn{3}{|l|}{ Adult populations } \\
\hline \multirow[t]{3}{*}{ Epidemiology } & Heterogeneity in transmission dynamics & Supplementary Table 1 \\
\hline & Role of asymptomatic/presymptomatic transmission & \\
\hline & Risk factors for severe disease outcomes & \\
\hline \multirow[t]{3}{*}{ Outbreak investigation } & Outbreak cessation & \\
\hline & Personnel and skill set & \\
\hline & Resources and tools & \\
\hline \multirow[t]{3}{*}{ Surveillance } & Detection strategies & \\
\hline & Population-level transmission risks & \\
\hline & Population surveillance & \\
\hline \multirow[t]{3}{*}{ Isolation precaution practices } & Initiation of precautions & Supplementary Table 2 \\
\hline & Management & \\
\hline & Discontinuation & \\
\hline \multirow[t]{3}{*}{ Personal protective equipment (PPE) } & Determination of appropriate PPE & \\
\hline & Strategies to improve PPE & \\
\hline & Impact on other viruses & \\
\hline \multirow[t]{3}{*}{ Environmental disinfection } & Risks related to environmental contamination & \\
\hline & Strategies for evaluating environmental contamination & \\
\hline & Disinfection strategies & \\
\hline \multirow[t]{3}{*}{ Shortages } & Impact of shortages & Supplementary Table 3 \\
\hline & Dissemination of best practices & \\
\hline & Clinical consequences & \\
\hline \multirow[t]{3}{*}{ Antimicrobial stewardship } & Healthcare utilization & \\
\hline & Coinfection & \\
\hline & Effective stewardship strategies & \\
\hline \multirow[t]{3}{*}{ Occupational safety } & HCP exposure & Supplementary Table 4 \\
\hline & HCP mental health & \\
\hline & Social/Organizational barriers and facilitators & \\
\hline \multirow[t]{3}{*}{ Return to work policies } & Risk in returning $\mathrm{HCP}$ & \\
\hline & Optimal criteria for return to work & \\
\hline & Sociocultural impact & \\
\hline \multirow[t]{6}{*}{ Pediatric populations } & Risk of disease transmission & Supplementary Table 5 \\
\hline & Estimate and mitigate transmission in schools & \\
\hline & $\begin{array}{l}\text { Long-term sequelae of symptomatic/asymptomatic disease } \\
\text { Risk factors for and long-term sequelae of MIS-C? }\end{array}$ & \\
\hline & Develop of effective screening algorithms to initiate isolation & \\
\hline & Significance of fecal shedding and environmental contamination & \\
\hline & Impact of COVID-19 on antibiotic prescribing? & \\
\hline
\end{tabular}

Note. HCP, healthcare personnel; PPE, personal protective equipment; MIS-C, multisystem inflammatory syndrome in children (MIS-C).

SARS-CoV-2 infection has had a disproportionate impact on racial and ethnic minority communities, frontline workers, and individuals with underlying conditions, such as diabetes, hypertension, obesity, and heart, lung, or kidney disease. ${ }^{7,18-21}$ Prioritizing research on the underlying societal and biological risk factors and optimal prevention and treatment for these high-risk groups is important. Furthermore, the extent and burden of long-term cardio-metabolic, respiratory, neurological, and psychological sequelae, including among asymptomatic individuals or those with mild disease, requires further study. ${ }^{8,22,23}$

\section{Outbreak investigation}

COVID-19 poses a unique challenge in outbreak investigation stemming from its novelty coupled with the rapid worldwide spread into all sectors of society, including into diverse healthcare 
settings in which patients, staff, and visitors can be the source of infection. As a result of asymptomatic transmission of uncertain route, COVID-19 requires a broad new approach to outbreak mitigation within healthcare settings, building upon the traditional public health methodologies. Many important potential avenues of research have opened in pursuit of this aim. ${ }^{24}$ Three research domains identified below (Supplementary Table 1 online) represent priority areas with unanswered questions about optimal COVID-19 outbreak investigations relevant to hospitals, nursing homes, and rehabilitation hospitals:

Priority area 1: Identify critical interventions required to stop COVID-19 outbreaks.

Priority area 2: Determine optimal personnel, expertise, and training required to conduct rapid SARS-CoV-2 and other outbreak investigations.

Priority area 3: Identify optimal resources and technology (reporting tools, software and hardware) to support outbreak investigations.

Several studies have highlighted the need for adequate resources, infrastructure, and personnel with expertise and leadership support to conduct timely, evidence-based infection prevention activities, including outbreak investigations. ${ }^{25-28} \mathrm{HCP}$ that work in overtaxed health care systems, faced with a rapidly spreading outbreak, as well as confusing and changing guidance, are at an elevated risk for burnout and moral distress. ${ }^{29}$ These challenges to conducting rapid and effective outbreak investigations are further amplified in nursing homes and small to mid-sized hospitals. ${ }^{30}$ Compared to larger hospitals, smaller hospitals face unique challenges, including infection preventionists (IPs) with other noninfection-related responsibilities, lack of specific IP training, lack of data synthesis and reporting tools, and high personnel turnover. $^{28,31-33}$ For example, rapid reporting systems can provide benchmarks to improve early outbreak detection in hospitals, nursing homes, and other healthcare settings leading to early interventions to curtail the outbreak. ${ }^{34}$ Technical knowhow and expertise in conducting outbreak investigations are important to identify key characteristics of the outbreak, including: populations being most affected; unique presentations that could vary by age, gender, race, comorbidities, or frailty; and patterns of transmission. Such expertise should also provide institutions with rapid, simple, systemic and culturally appropriate interventions.

\section{Surveillance strategies}

Robust surveillance of COVID-19 is critical to designing effective strategies for timely identification of COVID-19, limiting the spread of disease, and informing public health priorities and responses. Three research domains identified below (Supplementary Table 1 online) represent priority areas with unanswered questions in surveillance strategies relevant to healthcare and infection prevention:

Priority area 1: Determine optimal and rapid surveillance strategies to accurately define the scale and depth of COVID-19 and its impact on populations, communities, and individuals.

Priority area 2: Determine and evaluate high-yield, cost-effective, and efficient testing-based population surveillance strategies.

Priority area 3: Identify highest risk populations for targeted interventions based on their age, gender, race, comorbidities, settings, and community spread.
Reverse transcription polymerase chain reaction (RT-PCR) tests for SARS-CoV-2 can remain positive up to 3 months and do not directly translate to transmissibility. Viable virus has often not been found beyond 10 days in immunocompetent hosts, barring some instances. ${ }^{35-40}$ As a result, use of RT-PCR results for surveillance would overestimate COVID-19 incidence and prevalence, leading to misclassification of community-level burden. Large-scale longitudinal surveillance studies are needed to evaluate duration of test positivity (ie, RT-PCR, antigen, and serology) and risk for COVID-19 reinfection, with subgroup evaluation by symptoms (eg, asymptomatic, mild-to-moderate symptoms, and hospitalized patients). ${ }^{39,40}$

Although manufacturers report high sensitivity and specificity against assay controls, clinical sensitivity and specificity for COVID-19 infection is relatively unknown. In some instances, sensitivity has been reported to be as low as $70 \%$, depending on the quality of the specimen obtained and the time at which the sample is taken during a patient's illness. ${ }^{41,42}$ Studies are needed to evaluate the clinical performance characteristics of COVID-19 testing tools against the sensitivity and specificity of full-symptom screening, including early indicators of infection. Results from these studies will inform optimal sentinel surveillance strategies for large populations through en masse testing, such as pooled saliva sample testing or sewer line sampling.

Large-scale surveillance data within a wide variety of community and work settings and activities can lead to identification of groups and locations associated with high risk for transmission, leading to improved strategies for prevention and PPE use. Specific attention is needed within healthcare settings including nursing homes; assisted living facilities; group homes; factories and food processing plants; jails and prisons; and places of education such as schools, colleges, and universities. Supplementary Table 5 (online) highlights additional considerations relevant to pediatric surveillance, including surveillance for the multisystem inflammatory syndrome in children (MIS-C). Exposure risks may be further defined through novel surveillance tools (eg, personal exposure monitors and tracking apps).

\section{Isolation precaution practices}

Standard and transmission-based precaution practices are cornerstones of preventing transmission of infectious pathogens and ensuring HCP and patient safety across all healthcare settings. ${ }^{43}$ The US Centers for Disease Control and Prevention (CDC) developed and updated interim infection prevention and control recommendations regarding the use of transmission-based isolation precautions when caring for patients with suspected or confirmed SARS-CoV-2 infection in healthcare facilities. ${ }^{44}$ This guidance focuses on HCP and patient screening, testing protocols, patient placement and management practices, use of PPE, and family/visitor interactions.

However, as the COVID-19 pandemic continues to unfold, so does the need for a more rigorous evidence base to inform isolation practices and to assist healthcare facilities with effectively implementing public health guidance. Three research domains identified here (Supplementary Table 2 online) represent priority areas with unanswered questions in isolation precautions relevant to healthcare and infection prevention:

Priority area 1: Determine when and how to initiate transmissionbased isolation precautions for COVID-19.

Priority area 2: Determine how to optimize management and care delivery while isolation precautions are in place. 
Priority area 3: Determine when to discontinue COVID-19 isolation precautions and reinstitute isolation in cases of possible reinfection.

COVID-19 has a wide variety of clinical presentations ranging from asymptomatic to severely ill. ${ }^{45}$ Healthcare facilities use various criteria based on individual signs and symptoms to determine when to test individuals for COVID-19 and initiate isolation precautions while awaiting results, and they use various testing protocols to detect asymptomatic and presymptomatic individuals. Although these are critical strategies for stopping COVID-19 transmission, questions remain about the effectiveness of various screening and testing protocols to initiate isolation precautions practices and reduce transmission risk.

Once in isolation, use of PPE (ie, gloves, gown, mask, N95 respirator or power air-purifying respirator [PAPR], eye protection) for known or suspected COVID-19 patients can pose challenges to the delivery of care and can potentially delay recognition of other healthcare-associated conditions. ${ }^{46}$ Furthermore, COVID-19 isolation can be problematic for hospitalized patients and nursing home residents due to the use of equipment that can inhibit visual and auditory cues and visitor restrictions resulting in less family contact and support. The inability to connect with family members is one of the most distressing consequences of COVID-19 isolation, with a potential for long-lasting psychological consequences in survivors. ${ }^{47,48}$ Research to better understand, identify, and test approaches to mitigate the psychological, physical, and care delivery challenges related to COVID-19 isolation precaution practices, including the benefits and unintended consequences of family and visitor policies and restrictions, are needed. ${ }^{46-49}$

Recommendations for discontinuing isolation have been based on symptoms, test results, and time from positive test. ${ }^{37,50,51}$ Discontinuation of isolation precautions allows individuals to engage in normal and/or recovery-focused activities. To do so safely, however, discontinuation policies must also take into account the risk of secondary transmission. In other words, balancing risk of transmission events if isolation is discontinued too early against the risks of staying in isolation too long. Thus, research on when and how to safely discontinue isolation that balances public health and patient priorities is needed. Improved understanding of how continued positive test results correlate with transmission will also help guide isolation discontinuation policies. ${ }^{52}$

These research questions and resultant policy implications impact HCP directly. Early studies suggest that interventions, such as a triage committee and team decision making, may decrease the perception of personal culpability for untoward patient outcomes. ${ }^{29}$ Thus, meaningfully enhancing HCP engagement in developing research questions and in decision-making processes are critical to reducing moral distress and burnout.

\section{Personal protective equipment (PPE)}

General recommendations for HCP use of PPE are available from the CDC; expert groups have provided additional recommendations for use of PPE in crisis scenarios. ${ }^{5,44}$ Both CDC and expert guidance has been largely based on limited data extrapolated from other viral infections (eg, influenza, SARS-CoV-1) and/or studies with significant biases limiting generalizability. Research domains identified below (Supplementary Table 2 online) represent priority areas with unanswered questions concerning PPE use in healthcare settings:

Priority area 1: What is the appropriate level of universal PPE for current pandemic and afterward?
Priority area 2: What are the sociobehavioral, adaptive, and contextual factors required to improve appropriate PPE use?

Priority area 3: Are PPE interventions made during the COVID-19 pandemic likely to be effective against other commonly circulating respiratory viruses?

COVID-19 is generally thought to spread primarily through respiratory droplets; thus, the current role of PPE is aimed at decreasing droplet transmission. Masks are used as the cornerstone of source control (symptomatic or asymptomatic person with COVID-19). ${ }^{53}$ However, it is possible that COVID-19 transmission can occur through the eyes, either by direct droplet inoculation or via autoinoculation. In this setting, eye protection (face shields or goggles) has also been recommended and may play a key role in infection prevention. In a 2014 study that utilized a cough simulator and breathing worker simulator to model droplet transmission, face shields prevented exposure to droplets; however, masks were not used. ${ }^{54}$ A meta-analysis indicated that physical distancing, masks, and eye protection decreased the odds of COVID-19 transmission; however, the relative risk reduction of eye protection plus a face mask for COVID-19 has not been well described. ${ }^{55}$ Additionally, the benefit of face shields alone in source control of an asymptomatic or presymptomatic patient is not known.

Research on compliance with PPE guidance in prior outbreaks has focused on methods of delivering training. ${ }^{56}$ In the Ebola virus disease outbreak, a human-factors engineering approach to training and ensuring appropriate donning and doffing decreased ambiguity, explored failure modes, and enhanced teamwork to improve compliance with PPE guidance. ${ }^{57}$ Thus, other behavioral, adaptive, cultural, systemic or other factors may play a role in adherence to best-practice PPE use. Studies that utilize methods from healthcare epidemiology, infection prevention, human factors engineering, and medical sociology are needed to identify and mitigate (or enhance) sociobehavioral, adaptive, contextual, and human factors that impact appropriate PPE use. In the current pandemic, where mask-wearing has become politicized, understanding these factors may be even more essential. Understanding how to improve appropriate PPE use, through understanding the socio-behavioral, adaptive, and contextual reasons for not following appropriate PPE guidance, as well as human factors associated with appropriate PPE use, could improve PPE adherence among HCP. Finally, PPE use has been complicated by shortages, which resulted in institutions requiring healthcare providers to reuse single-use items such as $\mathrm{N}-95$ masks. The shortage of essential supplies can increase anxiety and fear in those who need them; however, the impact of PPE shortages on future use of PPE is a consideration that requires further investigation. ${ }^{58-60}$

\section{Environmental contamination and disinfection}

Surface contamination with SARS-CoV-2 has been frequently described, ${ }^{61-74}$ but the role of environmental contamination with SARS-CoV-2 in transmission in healthcare settings such as hospitals and nursing homes remains unclear. Three research domains identified below (Supplementary Table 2 online) represent priority areas with unanswered questions concerning environmental disinfection in healthcare settings:

Priority area 1: What are the risks associated with environmental contamination with SARS-CoV-2 for HCP and patients?

Priority area 2: What are optimized methods for identifying environmental contamination with SARS-CoV-2? 
Priority area 3: Determine the optimal methods for disinfection of healthcare environments.

Defining the risk associated with surface transmission is essential in assessing the potential benefit of decontamination and disinfection strategies. Although previously published studies demonstrate potential for fomite transmission, additional studies are needed to assess risk factors for both contamination and infection associated with contact with contaminated surfaces. In addition, these surface contamination studies were primarily cross-sectional studies and case reports using PCR detection of viral RNA on surfaces in COVID-19 units. Thus, while standardized methods have been proposed for evaluating surface contamination, evidence-based determination of optimal sampling strategies will enhance future work. ${ }^{75}$ Subsequent studies should include assessment of detection and correlation of findings with infectivity. Viral culture may be more useful in determining risk of infectivity, but there is inadequate infrastructure to broadly expand study of environmental contamination using this method. ${ }^{68}$ Enveloped viral surrogates including mammalian viruses and bacteriophage should be integrated into disinfection assessments. Establishing the infrastructure to define risk of surface contamination for other high-consequence pathogens is needed for future pandemic preparedness.

If surface transmission of COVID-19 is described, then reducing this risk within healthcare settings is necessary to provide care for vulnerable populations and to protect HCP. Implementation challenges can be substantial, but leadership support appears helpful. In a national study conducted in Thailand in 2014, Apisarnthanarak et $\mathrm{al}^{76}$ found that good-to-excellent hospital administration support for the infection control program was significantly associated with greater adherence to implemented environmental control and disinfection protocols. Thus, identifying the incremental benefit of enhanced disinfection strategies, such as ultraviolet germicidal irradiation, vaporized hydrogen peroxide, and others compared to commonly available disinfectants, will inform routine disinfection practices. ${ }^{72,77}$ The methods for evaluating the impact of such disinfection strategies should focus on clinical outcomes and laboratory methods that predict infectivity.

\section{Drug and medical supply-chain shortages}

The supply chain for drugs and medical supplies is global; most drugs and medical supplies are made outside the United States. ${ }^{78}$ Early in the pandemic, there were significant shortages of PPE, ventilators, and materials needed for laboratory detection of COVID-19. ${ }^{79}$ Drug shortages are situations in which patients are unable to access clinically interchangeable versions of regulated prescriptions due to supply limitations. ${ }^{78}$ Over the last decade, the number of drug shortages has increased dramatically. ${ }^{80,81}$ COVID-19 revealed consequences: manufacturers closed, governments prohibited drug and supply export, and patients and organizations stockpiled drugs. ${ }^{29,82-84}$ Of drugs consumed in the United States, $90 \%$ of raw active ingredients (active pharmaceutical ingredients [API]) are made in foreign facilities, $80 \%$ in China and India) ${ }^{78,85}$ Thus, supply-chain shortages are a complex global issue and can be influenced by geopolitical issues, trade, civil unrest, weather, and pandemics. ${ }^{82,86}$ The full extent of the impact of the COVID-19 pandemic, however, on the drug and medical supply chain is unknown. It is important to recognize and understand disruption in these supply chains to prepare for future pandemics and other global emergencies. Three research domains identified below
(Supplementary Table 3 online) represent priority areas with unanswered questions concerning drug and medical supply shortages:

Priority area 1: Define the extent of drug and medical supply chain shortages caused, directly or indirectly, by the COVID-19 pandemic.

Priority area 2: Identify methods to disseminate best practices in order to optimize patient care in the face of drug and medical supply chain shortages, from local protocols to international policies to help mitigate future shortages.

Priority area 3: Characterize clinical consequences of drug and medical supply-chain shortages.

Recognizing disruption that can occur as a result of drug and medical supply-chain disruption, legislation included in the March 2020 "Coronavirus Aid, Relief, and Economic Security (CARES) Act" requires drug manufacturers to report the anticipated duration and the problem leading to a shortage. ${ }^{80}$ However, studies on the extent of these disruptions and effectiveness of such policies during COVID-19 and after the pandemic is unknown.

Also unknown are the serious outcomes related to these shortages, including worsening illness and premature death. ${ }^{87-89}$ In addition, little is known about how drug shortages impact clinicians who prescribe medications to treat critically ill patients. HCP reported anxiety about their inability to provide competent and evidence-based care during COVID-19. ${ }^{60}$ Minority populations and vulnerable populations may be disproportionately affected by these shortages. Drugs and other supply shortages may pose ethical dilemmas when decisions must be made to treat one patient over another. ${ }^{90}$ The impact of shared decision making and triage committees, which remove the responsibility from the individual provider, on HCP mental health and resiliency should be systematically evaluated. ${ }^{29,60,90}$

Addressing these research questions related to supply-chain disruption and the impact of drug and medical supply shortages will help preparations for future global emergencies and will inform national and international policies aimed toward decreasing the impact of drug shortages on patient outcomes.

\section{Antimicrobial stewardship}

COVID-19 caused a rapid shift in the delivery of health care, including the suspension of elective procedures and transition of in-person visits to virtual encounters. ${ }^{91,92}$ Changing health care delivery may lead to an increase or decrease in antibiotic prescribing, depending on the setting and patient population. Three research domains identified below (Supplementary Table 3 online) represent priority areas with unanswered questions concerning antimicrobial stewardship in healthcare settings:

Priority area 1: Identify the impact of changes in healthcare utilization and delivery on antibiotic prescribing.

Priority area 2: Define epidemiology and risk factors related to bacterial and fungal coinfections in patients with COVID-19.

Priority area 3: Develop and implement optimal antimicrobial stewardship program (ASP) strategies to improve antimicrobial use and patient outcomes while adapting to changing healthcare delivery during COVID-19.

Although a decrease in the number of admitted patients may lead to a reduction in overall antimicrobial use, several studies have 
suggested a large percentage of COVID-19 patients presumptively receive antibiotics to treat the potential that the infection is bacterial or that a superimposed bacterial infection is leading to a greater severity of COVID-19. ${ }^{93-95}$ Similarly, changes in the volume of healthcare access (decreased hospitalizations and outpatient visits) during the pandemic will limit longitudinal comparisons due to altered denominators for typical use metrics, patient bed days of care (for acute care), and in-person visits (for outpatient care). Additionally, with routine pediatric care transitioning to predominantly telehealth visits, the pandemic's impact on outpatient antibiotic prescribing practices for children remain unexamined (Supplementary Table 5 online). Finally, the downstream impact of changes in antimicrobial prescribing on antimicrobial resistance and Clostridium difficile are unknown.

Typically viral respiratory infections have been associated with an increased risk of bacterial or fungal coinfections, which substantially increases risks of morbidity and mortality. ${ }^{96-98}$ However, data on coinfections in COVID-19 have been sparse and heterogeneous, with estimates ranging from $3 \%$ to $30 \% .{ }^{99-108}$ To inform antimicrobial treatment, we must understand risk factors and timing for the development of coinfection. For example, the risk of coinfection related to comorbidity (eg, immunosuppression) or exposure (eg, hospitalization, ventilation, device-placement) is unknown. ${ }^{97}$ Furthermore, quantification of coinfection is limited by diagnostic difficulties including (1) distinguishing colonization from infection; (2) improving diagnosis of coinfection versus alternative causes of decompensation (eg, acute respiratory distress syndrome from COVID-19); and (3) limited respiratory culture data due to SARS-CoV-2 transmission concerns. ${ }^{109}$

The ongoing COVID-19 pandemic has drastically changed the way that ASP teams interact with patients and other healthcare providers. ${ }^{110,111}$ Although the focus of ASPs may have shifted during the pandemic, improving antimicrobial use and stemming the tide of the development of antibiotic resistance remain at the core. ${ }^{110,111}$ ASP teams are now ubiquitous in US hospitals, but ensuring that all hospitals have adequate infectious disease expertise on their teams may require novel approaches. ${ }^{112}$ Simultaneously, the responsibilities of ASPs have increasedwith many playing an active role in COVID-19 management, such as guideline development or remdesivir allocation. ${ }^{13,114}$ Virtual strategies, such as 'tele-stewardship' and nimble regional or national 'hotlines,' particularly for small and rural hospitals, need to be evaluated and implemented. Likewise, although some nursing homes have established comprehensive nursing home ASPs, a required condition of participation by the US Centers for Medicare and Medicaid Services (CMS), many would benefit from additional support. ${ }^{95}$

\section{Healthcare personnel safety and occupational safety}

The COVID-19 pandemic has raised concerns about HCP safety. Research is needed to identify strategies to protect HCP from acquiring SARS-CoV-2 at work and to support HCP from physical, psychological, social, and organizational challenges related to the pandemic. ${ }^{58,59,76}$ Three research domains identified below (Supplementary Table 4 online) represent priority areas with unanswered questions concerning HCP and occupational safety:

Priority area 1: Define risks that increase HCP exposure to and acquisition of SARS-CoV-2 and interventions that can mitigate these risks.
Priority area 2: Determine optimized strategies to protect HCP emotional and psychological health.

Priority area 3: Determine impact of social and organizational strategies to maintain the health and wellness of HCP.

Understanding the factors that increase HCP risk of acquiring COVID-19 is essential to develop an evidence-based infection prevention program. These factors may include attributes of the patients under the HCP's care (eg, clinical symptoms, comorbid conditions), aspects of the care delivered (eg, procedures performed, duration of contact, number of patients under their care during a shift, preoperative screening), HCP practices (eg, PPE utilized, years of experience), and work site (eg, leadership support, control over practice). In addition, understanding individual HCP factors that increase the likelihood of an infected HCP developing more severe disease and adverse outcomes will help determine which HCP may need additional protections in place, such as furlough, reassignment from the care of COVID-19 patients, or ongoing testing at a set interval during hospitalization. Research on costs and effectiveness of policies, such as preoperative and on-admission screening, can inform practice, improve HCP safety, enhance HCP confidence in safety processes, and reduce staffing challenges. ${ }^{29}$

Controversy persists about determining what medical procedures may allow opportunistic airborne transmission of pathogens traditionally considered to follow droplet transmission. Key considerations include the size of particles generated during specific procedures, and the ability of the pathogen to survive in small particles, and the infectious dose for the pathogen (amount of virus carried in small airborne particles sufficient to cause infection if inhaled). These questions have important implications for air handling and PPE selection recommendations to minimize transmission risk in healthcare settings.

Identifying policies that support the social, emotional, and economic needs of HCP is critical to maintaining workforce resilience and decreasing presenteeism, burnout, and turnover during the protracted COVID-19 pandemic. ${ }^{60}$ Developing an evidence base to inform these policies requires understanding how the pandemic has affected HCP social, emotional, and physical health, finances, ability to care for families, and decisions to come to work. A mixedmethods implementation science approach can inform local, institutional, and national policies and practices to support HCP resiliency, job security, ability to isolate or quarantine effectively, and social support (eg, hazard and sick pay policies, housing options, and consistent childcare). ${ }^{106}$

\section{Return to work}

Recommendations for return to work following COVID-19 infection are largely derived from experience with other communicable diseases such as influenza and norovirus. COVID-19 has posed specific challenges in timely return to work strategies due to minimal data on the true transmission dynamics and nature of exposure risks during $\mathrm{HCP}$-patient or $\mathrm{HCP}$-coworker interactions. Little is known about which HCP roles or activities confer the highest risks of transmission and how this risk is modified by severity of prior illness or presence of lingering symptoms upon return. Three research domains identified below (Supplementary Table 4 online) address the urgent priorities for research to facilitate timely worker return while tempering the risks of premature return to work during active illness: ${ }^{115}$ 
Priority area 1: Determine risk of SARS-CoV-2 transmission by returning $\mathrm{HCPs}$ to coworkers and patients, by HCP type and setting.

Priority area 2: Determine the optimal criteria and modifications necessary for earliest safe return to work.

Priority area 3: Determine the sociocultural impact of and strategies for successful return-to-work for HCP.

As of November 2020, the US CDC recommends that HCP who have had COVID-19 return at 10 days from initial symptom onset (20 days if immunocompromised) if improved and fever-free for 24 hours without fever-reducing medications. ${ }^{116}$ During a pandemic, sick leave of this duration, even when asymptomatic, could significantly impact healthcare system staffing. Many employers are considering the use of test-based strategies to shorten the window for HCP to return to work. Shared anecdotal experiences suggest a broad range of approaches for return to work across institutions and locations (eg, length of furloughs, strategies to address persistently PCR-positive recovered workers, coworker education, and acculturation to mitigate social stigma).

The true risk of virus transmission to coworkers or others from asymptomatic or minimally symptomatic HCP to patients or other coworkers remains largely unknown. Research is needed to assess the relative importance of worker-related factors, such as viral viability in minimally symptomatic or immunocompromised HCP, effectiveness of PPE or physical barriers to mitigate transmission, and risk of acquisition among immunocompromised coworkers and patients. HCP workflow, social culture, and nature of coworker interactions may also affect the likelihood of transmission within the workplace. Finally, research addressing worker reintegration and actions to assuage social stigmas (eg, educational needs of workers, childcare) are vital to retaining a talented and prepared workforce. ${ }^{90}$

\section{Conclusion}

The SHEA COVID-19 research agenda is critical and ambitious. COVID-19 has exposed dangerous gaps in our understanding of the epidemiology, transmission, and individual as well as public health consequences of viral diseases. Global impacts on health, the economy, and progress have been felt in every population and country. The disease has disproportionately affected older adults, especially those living in nursing homes or long-term care facilities, racial minorities, and those with multiple comorbidities. Supply shortages have affected the health and well-being of HCP and have negatively affected care of those not infected with COVID-19. A well-planned, collaborative, comprehensive research agenda with careful, dedicated, and timely execution is a critical element to address the most important questions to more effectively limit outbreaks and pandemics. With the recognition that pandemics do not respect boundaries or economies, close collaboration between various disciplines is crucial. Research initiatives and trust between industrialized and developing nations are needed to address these critical questions in ways generalizable around the globe, including attention to capacity building, technology transfer, training resources, and aligning surveillance and prevention activities. This research agenda is a snapshot in time during the worst days of the COVID-19 pandemic and will certainly shift as the pandemic evolves and as vaccines and other therapeutics become available. Nonetheless, several priorities outlined have relevance to future infectious disease outbreaks and epidemics.
This research agenda calls for translational studies from laboratory-based basic science research to well-designed, largescale studies and health outcomes research. To undertake this work, funding organizations must make COVID-19 research their highest priority. We anticipate that the next decade will be crucial in developing the next generation of epidemiologists, IPs, researchers, and leaders.

Supplementary material. To view supplementary material for this article, please visit https://doi.org/10.1017/ice.2021.25

Acknowledgments. The authors thank Ms Lynne Batshon (Society for Healthcare Epidemiology), Mr John Heys (Society for Healthcare Epidemiology), Mr Jason Engle (Michigan Medicine) and Ms Kristen Gibson (Michigan Medicine) for their commitment, project management, editing, and formatting assistance in preparing this research agenda.

Financial support. No financial support was provided relevant to this article.

Conflicts of interest. All authors report no conflicts of interest relevant to this article.

\section{References}

1. Teixeira da Silva JA, Tsigaris P, Erfanmanesh M. Publishing volumes in major databases related to COVID-19. Scientometrics 2020:1-12.

2. Bhimraj A, Morgan RL, Shumaker AH, et al. Infectious Diseases Society of America guidelines on the treatment and management of patients with COVID-19. Infectious Diseases Society of America website. https:// www.idsociety.org/practice-guideline/covid-19-guideline-treatment-andmanagement/. Published 2020. Accessed October 16, 2020.

3. Hanson KE, Caliendo AM, Arias CA, et al. Infectious Diseases Society of America guidelines on the diagnosis of COVID-19: serologic testing. Infectious Diseases Society of America website. https://www.idsociety. org/practice-guideline/covid-19-guideline-serology/. Published 2020. Accessed October 16, 2020.

4. Hanson KE, Caliendo AM, Arias CA, et al. Infectious Diseases Society of America guidelines on the diagnosis of COVID-19. Infectious Diseases Society of America website. 2020. https://www.idsociety.org/practiceguideline/covid-19-guideline-diagnostics/. Accessed October 16, 2020.

5. Lynch JB, Davitkov P, Anderson DJ, et al. Infectious Diseases Society of America guidelines on infection prevention in patients with suspected or known COVID-19. Infectious Diseases Society of America website. https://www.idsociety.org/practice-guideline/covid-19-guideline-infectionprevention/. Published 2020. Accessed October 16, 2020.

6. Beldomenico PM. Do superspreaders generate new superspreaders? A hypothesis to explain the propagation pattern of COVID-19. Int $J$ Infect Dis 2020;96:461-463.

7. Mills JP, Kaye KS, Mody L. COVID-19 in older adults: clinical, psychosocial, and public health considerations. JCI Insight 2020;5(10):e139292.

8. Ouslander JG, Grabowski DC. COVID-19 in nursing homes: calming the perfect storm. J Am Geriatr Soc 2020;68:2153-2162.

9. Calderwood MS, Deloney VM, Anderson DJ, et al. Policies and practices of SHEA Research Network hospitals during the COVID-19 pandemic. Infect Control Hosp Epidemiol 2020;41:1127-1135.

10. Day M. COVID-19: identifying and isolating asymptomatic people helped eliminate virus in Italian village. BMJ 2020;368:m1165.

11. Ellul M, Benjamin L, Singh B, et al. Neurological Associations of COVID-19. Lancet Neurol 2020;19:767-783.

12. Gudbjartsson DF, Helgason A, Jonsson H, et al. Spread of SARS-CoV-2 in the Icelandic Population. N Engl J Med 2020;382:2302-2315.

13. Killerby ME, Link-Gelles R, Haight SC, et al. Characteristics associated with hospitalization among patients with COVID-19-metropolitan Atlanta, Georgia, March-April 2020. Morb Mortal Wkly Rep 2020;69: 790-794.

14. Kim L, Whitaker M, O'Halloran A, et al. Hospitalization rates and characteristics of children aged $<18$ years hospitalized with laboratoryconfirmed COVID-19-COVID-NET, 14 States, March 1-July 25, 2020. Morb Mortal Wkly Rep 2020;69:1081-1088. 
15. Kim L, Garg S, O'Halloran A, et al. Risk factors for intensive care unit admission and in-hospital mortality among hospitalized adults identified through the US Coronavirus Disease 2019 (COVID-19)-Associated Hospitalization Surveillance Network (COVID-NET). Clin Infect Dis 2020. doi: 10.1093/cid/ciaa1012.

16. Kimball A, Hatfield KM, Arons M, et al. Asymptomatic and presymptomatic SARS-CoV-2 infections in residents of a long-term care skilled nursing facility - King County, Washington, March 2020. Morb Mortal Wkly Rep 2020;69:377-381.

17. Lee S, Kim T, Lee E, et al. Clinical course and molecular viral shedding among asymptomatic and symptomatic patients with SARS-CoV-2 Infection in a community treatment center in the Republic of Korea. JAMA Intern Med 2020;180:1447-1452.

18. Lian J, Jin X, Hao S, et al. Analysis of epidemiological and clinical features in older patients with coronavirus disease 2019 (COVID-19) outside Wuhan. Clin Infect Dis 2020;71:740-747.

19. Liu Y, Eggo RM, Kucharski AJ. Secondary attack rate and superspreading events for SARS-CoV-2. Lancet 2020;395:e47.

20. Mizumoto K, Kagaya K, Zarebski A, Chowell G. Estimating the asymptomatic proportion of coronavirus disease 2019 (COVID-19) cases on board the Diamond Princess cruise ship, Yokohama, Japan, 2020. Euro Surveill 2020;25(10):2000180.

21. Nishiura H, Kobayashi T, Miyama T, et al. Estimation of the asymptomatic ratio of novel coronavirus infections (COVID-19). Int J Infect Dis 2020;94:154-155.

22. Prescott HC, Sussman JB, Wiersinga WJ. Postcritical illness vulnerability. Curr Opin Crit Care 2020;26:500-507.

23. Puntmann VO, Carerj ML, Wieters I, et al. Outcomes of cardiovascular magnetic resonance imaging in patients recently recovered from coronavirus disease 2019 (COVID-19). JAMA Cardiol 2020;5:1265-1273.

24. Principles of epidemiology in public health practice. Lesson 6: investigating an outbreak. US Centers for Disease Control and Prevention website. https://www.cdc.gov/csels/dsepd/ss1978/lesson6/section2.html. Published 2016. Accessed October 16, 2020.

25. Stone PW, Dick A, Pogorzelska M, Horan TC, Furuya EY, Larson E. Staffing and structure of infection prevention and control programs. Am J Infect Control 2009;37:351-357.

26. Saint S, Greene MT, Fowler KE, et al. What US hospitals are currently doing to prevent common device-associated infections: results from a national survey. BMJ Qual Saf 2019;28:741-749.

27. Hessels AJ, Kelly AM, Chen L, Cohen B, Zachariah P, Larson EL. Impact of infectious exposures and outbreaks on nurse and infection preventionist workload. Am J Infect Control 2019;47:623-627.

28. Herzig CT, Stone PW, Castle N, Pogorzelska-Maziarz M, Larson EL, Dick AW. Infection prevention and control programs in US nursing homes: results of a national survey. J Am Med Dir Assoc 2016;17:85-88.

29. Borges LM, Barnes SM, Farnsworth JK, Bahraini NH, Brenner LA. A commentary on moral injury among health care providers during the COVID19 pandemic. Psychol Trauma 2020;12:S138-S140.

30. Montoya A, Jenq G, Mills JP, et al. Partnering with local hospitals and public health to manage COVID-19 outbreaks in nursing homes. J Am Geriatr Soc 2021;69:30-36.

31. Mody L, Langa KM, Saint S, Bradley SF. Preventing infections in nursing homes: a survey of infection control practices in southeast Michigan. Am J Infect Control 2005;33:489-492.

32. Rhinehart E, Walker S, Murphy D, O'Reilly K, Leeman P. Frequency of outbreak investigations in US hospitals: results of a national survey of infection preventionists. Am J Infect Control 2012;40:2-8.

33. Santibanez S, Bellis KS, Bay A, et al. Strengthening rural states' capacity to prepare for and respond to emerging infectious diseases, 2013-2015. South Med J 2019;112:101-105.

34. Cheng VC, Tai JW, Wong LM, et al. Effect of proactive infection control measures on benchmarked rate of hospital outbreaks: an analysis of public hospitals in Hong Kong over 5 years. Am J Infect Control 2015;43:965-970.

35. Criteria for releasing COVID-19 patients from isolation. 2020. World Health Organization website. https://apps.who.int/iris/handle/10665/ 332451. Accessed November 13, 2020.
36. Duration of Isolation and Precautions for Adults with COVID-19. US Centers for Disease Control and Prevention website. https://www.cdc. gov/coronavirus/2019-ncov/hcp/duration-isolation.html. Published 2020. Accessed November 11, 2020.

37. van Kampen J, van de Vijver D, Fraaij P, et al. Shedding of infectious virus in hospitalized patients with coronavirus disease-2019 (COVID19): duration and key determinants. 2020. medRxiv June 8, 2020. doi: 10.1101/2020. 06.08.20125310v1.

38. Wolfel R, Corman VM, Guggemos W, et al. Virological assessment of hospitalized patients with COVID-2019. Nature 2020;581:465-469.

39. Bullard J, Dust K, Funk D, et al. Predicting infectious SARS-CoV-2 from diagnostic samples. Clin Infect Dis 2020. doi: 10.1093/cid/ciaa638.

40. Basile K, McPhie K, Carter I, et al. Cell-based culture of SARS-CoV-2 informs infectivity and safe de-isolation assessments during COVID19. Clin Infect Dis 2020. doi: 10.1093/cid/ciaa1579.

41. Yang Y, Yang M, Shen C, et al. Evaluating the accuracy of different respiratory specimens in the laboratory diagnosis and monitoring the viral shedding of 2019-nCoV infections. medRxiv February 11, 2020. doi: 10.1101/2020.02.11.20021493v2.

42. Kucirka LM, Lauer SA, Laeyendecker O, Boon D, Lessler J. Variation in falsenegative rate of reverse transcriptase polymerase chain reaction-based SARSCoV-2 tests by time since exposure. Ann Intern Med 2020;173:262-267.

43. Siegel J, Rhinehart E, Jackson M, Chiarello L, Healthcare Infection Control Practices Advisory Committee. Guideline for isolation precautions: preventing transmission of infectious agents in healthcare settings (2007). Centers for Disease Control and Prevention website. https://www.cdc. gov/infectioncontrol/guidelines/isolation/index.html. Published 2007. Accessed October 16, 2020.

44. Interim infection prevention and control recommendations for healthcare personnel during the coronavirus disease 2019 (COVID-19) pandemic. Centers for Disease Control and Prevention website. https://www.cdc. gov/coronavirus/2019-ncov/hcp/infection-control-recommendations.html. Published 2020. Accessed October 16, 2020.

45. National Center for Immunization and Respiratory Diseases (NCIRD) Division of Viral Diseases. Symptoms of coronavirus. Centers for Disease Control and Prevention website. https://www.cdc.gov/coronavirus/2019ncov/symptoms-testing/symptoms.html. Published 2020. Accessed November 2, 2020.

46. Reyes R, Bono G, T EF. So-called urinary tract infection in the era of COVID-19. J Am Geriatr Soc 2020;68:1927-1928.

47. Annweiler C, Sacco G, Salles N, et al. National French survey of COVID19 symptoms in people aged 70 and over. Clin Infect Dis 2020. doi: 10. 1093/cid/ciaa792.

48. O'Hanlon S, Inouye SK. Delirium: a missing piece in the COVID-19 pandemic puzzle. Age Ageing 2020;49:497-498.

49. Verbeek H, Gerritsen DL, Backhaus R, de Boer BS, Koopmans R, Hamers JPH. Allowing visitors back in the nursing home during the COVID-19 crisis: a Dutch national study into first experiences and impact on wellbeing. J Am Med Dir Assoc 2020;21:900-904.

50. National Centre for Infectious Diseases and the Chapter of Infectious Disease Physicians. Period of infectivity to inform strategies for de-isolation for COVID-19 patients. Academy of Medicine - Singapore website. https://www.ams.edu.sg/view-pdf.aspx?file=media\%5c5556_fi_331.pdf\& ofile $=$ Period + of + Infectivity + Position + Statement $+($ final $)+23-5-20+$ (logos).pdf. Published 2020. Accessed October 16, 2020.

51. Discontinuation of transmission-based precautions and disposition of patients with COVID-19 in healthcare settings (interim guidance). Centers for Disease Control and Prevention website. https://www.cdc. gov/coronavirus/2019-ncov/hcp/disposition-hospitalized-patients.html. Published 2020. Accessed October 16, 2020.

52. Korean Disease Control and Prevention Agency, Division of Risk Assessment and International Cooperation. Findings from investigation and analysis of re-positive cases. Korean Disease Control and Prevention Agency website. https://www.cdc.go.kr/board/board.es? $\mathrm{mid}=$ a30402000000\&bid=0030. Published 2020. Accessed October 16, 2020.

53. Cheng VC, Wong SC, Chuang VW, et al. The role of community-wide wearing of face mask for control of coronavirus disease 2019 (COVID-19) epidemic due to SARS-CoV-2. J Infect 2020;81:107-114. 
54. Lindsley WG, Noti JD, Blachere FM, Szalajda JV, Beezhold DH. Efficacy of face shields against cough aerosol droplets from a cough simulator. $J$ Occup Environ Hyg 2014;11:509-518.

55. Chu DK, Akl EA, Duda S, et al. Physical distancing, face masks, and eye protection to prevent person-to-person transmission of SARS-CoV-2 and COVID-19: a systematic review and meta-analysis. Lancet 2020;395: 1973-1987.

56. Verbeek JH, Rajamaki B, Ijaz S, et al. Personal protective equipment for preventing highly infectious diseases due to exposure to contaminated body fluids in healthcare staff. Cochrane Database Syst Rev 2020;5: CD011621.

57. Gurses AP, Rosen MA, Pronovost PJ. Improving guideline compliance and healthcare safety using human factors engineering: the case of Ebola. J Patient Saf Risk Manage 2018;23:93-95.

58. Apisarnthanarak A, Apisarnthanarak P, Siripraparat C, Saengaram P, Leeprechanon N, Weber DJ. Impact of anxiety and fear for COVID-19 toward infection control practices among Thai healthcare workers. Infect Control Hosp Epidemiol 2020;41:1093-1094.

59. Civantos AM, Byrnes Y, Chang C, et al. Mental health among otolaryngology resident and attending physicians during the COVID-19 pandemic: national study. Head Neck 2020;42:1597-1609.

60. Shanafelt T, Ripp J, Trockel M. Understanding and addressing sources of anxiety among health care professionals during the COVID-19 pandemic. JAMA 2020;323:2133-2134.

61. Cheng VC, Wong SC, Chan VW, et al. Air and environmental sampling for SARS-CoV-2 around hospitalized patients with coronavirus disease 2019 (COVID-19). Infect Control Hosp Epidemiol 2020;41:1258-1265.

62. Chia PY, Coleman KK, Tan YK, et al. Detection of air and surface contamination by SARS-CoV-2 in hospital rooms of infected patients. Nat Commun 2020;11:2800.

63. Guo ZD, Wang ZY, Zhang SF, et al. Aerosol and surface distribution of severe acute respiratory syndrome coronavirus 2 in hospital wards, Wuhan, China, 2020. Emerg Infect Dis 2020;26:1583-1591.

64. Lee SE, Lee DY, Lee WG, et al. Detection of novel coronavirus on the surface of environmental materials contaminated by COVID-19 patients in the Republic of Korea. Osong Public Health Res Perspect 2020;11: $128-132$.

65. Lei H, Ye F, Liu X, et al. SARS-CoV-2 environmental contamination associated with persistently infected COVID-19 patients. Influenza Other Respir Viruses 2020;14:688-699.

66. Li YH, Fan YZ, Jiang L, Wang HB. Aerosol and environmental surface monitoring for SARS-CoV-2 RNA in a designated hospital for severe COVID-19 patients. Epidemiol Infect 2020;148:e154.

67. Ong SWX, Tan YK, Chia PY, et al. Air, Surface environmental, and personal protective equipment contamination by severe acute respiratory syndrome coronavirus 2 (SARS-CoV-2) from a symptomatic patient. JAMA 2020;323:1610-1612.

68. Pasquarella C, Colucci ME, Bizzarro A, et al. Detection of SARS-CoV-2 on hospital surfaces. Acta Biomed 2020;91:76-78.

69. Razzini K, Castrica M, Menchetti L, et al. SARS-CoV-2 RNA detection in the air and on surfaces in the COVID-19 ward of a hospital in Milan, Italy. Sci Total Environ 2020;742:140540.

70. Ryu BH, Cho Y, Cho OH, Hong SI, Kim S, Lee S. Environmental contamination of SARS-CoV-2 during the COVID-19 outbreak in South Korea. Am J Infect Control 2020;48:875-879.

71. Zhou Y, Zeng Y, Chen C. Presence of SARS-CoV-2 RNA in isolation ward environment 28 days after exposure. Int J Infect Dis 2020;97:258-259.

72. Zhou J, Otter JA, Price JR, et al. Investigating SARS-CoV-2 surface and air contamination in an acute healthcare setting during the peak of the COVID-19 pandemic in London. Clin Infect Dis 2020. doi: 10.1093/ cid/ciaa905.

73. Wu S, Wang Y, Jin X, Tian J, Liu J, Mao Y. Environmental contamination by SARS-CoV-2 in a designated hospital for coronavirus disease 2019. Am J Infect Control 2020;48:910-914.

74. Ye G, Lin H, Chen S, et al. Environmental contamination of SARS-CoV-2 in healthcare premises. J Infect 2020. doi: 10.1016/j.jinf.2020.04.034.

75. Surface sampling of coronavirus disease (COVID-19): a practical "how to" protocol for health care and public health professionals. World Health
Organization website. https://apps.who.int/iris/rest/bitstreams/1269401/ retrieve. Published 2020. Accessed January 2021.

76. Apisarnthanarak A, Weber DJ, Ratz D, Saint S, Khawcharoenporn T, Greene MT. National survey of environmental cleaning and disinfection in hospitals in Thailand. Infect Control Hosp Epidemiol 2017;38: 1250-1253.

77. Ye G, Lin $\mathrm{H}$, Chen S, et al. Environmental contamination of SARS-CoV-2 in healthcare premises. J Infect 2020;81:e1-e5.

78. US Food and Drug Administration and Duke Margolis Center for Health Policy. Identifying the root causes of drug shortages and finding enduring solutions (public meeting and request for comments). https://www. regulations.gov/document?D=FDA-2018-N-3272-0001. Published 2018. Accessed October 16, 2020.

79. Medical device shortages during the COVID-19 public health emergency. US Food and Drug Administration website. https://www.fda.gov/medicaldevices/coronavirus-covid-19-and-medical-devices/medical-deviceshortages-during-covid-19-public-health-emergency. Published 2020. Accessed November 2, 2020.

80. US Congress. S.3548-CARES Act. US Congress website. https://www. congress.gov/bill/116th-congress/senate-bill/3548. Published 2020. Accessed November 13, 2020.

81. Balfour H. COVID-19 update: coronavirus and the pharmaceutical supply chain. European Pharmacy Review website. https://www. europeanpharmaceuticalreview.com/article/116145/covid-19-updatecoronavirus-and-the-pharmaceutical-supply-chain/. Published 2020. Accessed October 16, 2020.

82. Bruinen de Bruin Y, Lequarre AS, McCourt J, et al. Initial impacts of global risk mitigation measures taken during the combatting of the COVID-19 pandemic. Saf Sci 2020;128:104773.

83. Chatterjee P. Indian pharma threatened by COVID-19 shutdowns in China. Lancet 2020;395:675.

84. De Weerdt E, Simoens S, Casteels M, Huys I. Clinical, economic and policy implications of drug shortages in the European Union. Appl Health Econ Health Policy 2017;15:441-445.

85. US Food and Drug Administration and Duke Margolis Center for Health Policy. Identifying the root causes of drug shortages and finding enduring solutions (discussion guide): improving the efficiency of outcome validation in the Sentinel system. Duke University website. https://healthpolicy. duke.edu/sites/default/files/2020-02/discussion_guide.pdf. Published 2018. Accessed November 12, 2020.

86. Wang M, Jie F. Managing supply chain uncertainty and risk in the pharmaceutical industry. Health Serv Manage Res 2020;33:156-164.

87. Gray A, Manasse Jr HR. Shortages of medicines: a complex global challenge. SciELO Public Health 2012. doi: 10.2471/BLT.11.101303.

88. Metzger ML, Billett A, Link MP. The impact of drug shortages on children with cancer-the example of mechlorethamine. N Engl J Med 2012; 367:2461-2463.

89. Phuong JM, Penm J, Chaar B, Oldfield LD, Moles R. The impacts of medication shortages on patient outcomes: a scoping review. PLoS One 2019;14:e0215837.

90. Shale S. Moral injury and the COVID-19 pandemic: reframing what it is, who it affects and how care leaders can manage it. BMJ Leader 2020. doi: 10.1136/leader-2020-000295.

91. Chen F, Sun W, Sun S, Li Z, Wang Z, Yu L. Clinical characteristics and risk factors for mortality among inpatients with COVID-19 in Wuhan, China. Clin Transl Med 2020. doi: 10.1002/ctm2.40.

92. Chen N, Zhou M, Dong X, et al. Epidemiological and clinical characteristics of 99 cases of 2019 novel coronavirus pneumonia in Wuhan, China: a descriptive study. Lancet 2020;395:507-513.

93. Clancy CJ, Nguyen MH. Coronavirus disease 2019, superinfections, and antimicrobial development: what can we expect? Clin Infect Dis 2020. doi: $10.1093 /$ cid/ciaa524.

94. Davis MW, McManus D, Koff A, et al. Repurposing antimicrobial stewardship tools in the electronic medical record for the management of COVID-19 patients. Infect Control Hosp Epidemiol 2020;41:1335-1337.

95. Fu CJ, Mantell E, Stone PW, Agarwal M. Characteristics of nursing homes with comprehensive antibiotic stewardship programs: results of a national survey. Am J Infect Control 2020;48:13-18. 
96. Hartnett KP. Impact of the COVID-19 Pandemic on Emergency Department Visits-United States, January 1, 2019-May 30, 2020. Morbid Mortal Wkly Rep 2020;69:699-704.

97. Hughes S, Troise O, Donaldson H, Mughal N, Moore L. Bacterial and fungal coinfection among hospitalised patients with COVID-19: a retrospective cohort study in a UK secondary care setting. Clin Microbiol Infect 2020;26:1395-1399.

98. Klein EY, Monteforte B, Gupta A, et al. The frequency of influenza and bacterial coinfection: a systematic review and meta-analysis. Influenza Other Respir Viruses 2016;10:394-403.

99. Huttner B, Catho G, Pano-Pardo JR, Pulcini C, Schouten J. COVID-19: don't neglect antimicrobial stewardship principles! Clin Microbiol Infect 2020;26:808-810.

100. Langford BJ, So M, Raybardhan S, et al. Bacterial co-infection and secondary infection in patients with COVID-19: a living rapid review and metaanalysis. Clin Microbiol Infect 2020;26:1622-1629.

101. MacIntyre CR, Chughtai AA, Barnes M, et al. The role of pneumonia and secondary bacterial infection in fatal and serious outcomes of pandemic influenza a(H1N1)pdm09. BMC Infect Dis 2018;18:637.

102. Morens DM, Taubenberger JK, Fauci AS. Predominant role of bacterial pneumonia as a cause of death in pandemic influenza: implications for pandemic influenza preparedness. J Infect Dis 2008;198:962-970.

103. Nestler M, Godbout E, Lee K, et al. Impact of COVID-19 on pneumoniafocused antibiotic use at an academic medical center. Infect Control Hosp Epidemiol 2020. doi: 10.1017/ice.2020.362.

104. Nori P, Cowman K, Chen V, et al. Bacterial and fungal co-infections in COVID-19 patients hospitalized during the New York City pandemic surge. Infect Control Hosp Epidemiol 2020. doi: 10.1017/ice. 2020.368.

105. Nori P, Szymczak W, Puius Y, et al. Emerging co-pathogens: New Delhi metallo-beta-lactamase-producing Enterobacterales infections in New York City COVID-19 patients. Int J Antimicrob Agents 2020;5(6):106179.

106. PeConga EK, Gauthier GM, Holloway A, et al. Resilience is spreading: mental health within the COVID-19 pandemic. Psychol Trauma 2020;12:S47-S48.
107. Beumer MC, Koch RM, van Beuningen D, et al. Influenza virus and factors that are associated with ICU admission, pulmonary co-infections and ICU mortality. J Crit Care 2019;50:59-65.

108. Shah MM, Hsiao EI, Kirsch CM, Gohil A, Narasimhan S, Stevens DA. Invasive pulmonary aspergillosis and influenza co-infection in immunocompetent hosts: case reports and review of the literature. Diagn Microbiol Infect Dis 2018;91:147-152.

109. Vaughn VM, Gandhi T, Petty LA, et al. Empiric antibacterial therapy and community-onset bacterial co-infection in patients hospitalized with COVID-19: a multihospital cohort study. Clin Infect Dis 2020. doi: 10. 1093/cid/ciaa1239.

110. Stevens MP, Patel PK, Nori P. Involving antimicrobial stewardship programs in COVID-19 response efforts: all hands on deck. Infect Control Hosp Epidemiol 2020;41:744-745.

111. Spernovasilis N, Kofteridis D. COVID-19 and antimicrobial stewardship: What is the interplay? Infect Control Hosp Epidemiol 2020. doi: 10.1017/ ice.2020.246.

112. Vaughn VM, Greene MT, Ratz D, et al. Antibiotic stewardship teams and Clostridioides difficile practices in United States hospitals: a national survey in The Joint Commission antibiotic stewardship standard era. Infect Control Hosp Epidemiol 2020;41:143-148.

113. Stevens MP, Patel PK, Nori P. Antimicrobial stewardship programs and convalescent plasma for COVID-19: a new paradigm for preauthorization? Infect Control Hosp Epidemiol 2020. doi: 10.1017/ice.2020.459.

114. Mazdeyasna $\mathrm{H}$, Nori $\mathrm{P}$, Patel $\mathrm{P}$, et al. Antimicrobial stewardship at the core of COVID-19 response efforts: implications for sustaining and building programs. Curr Infect Dis Rep 2020;22:23.

115. Tartari E, Saris K, Kenters N, et al. Not sick enough to worry? "Influenzalike" symptoms and work-related behavior among healthcare workers and other professionals: Results of a global survey. PLoS One 2020;15: e0232168.

116. Criteria for return to work for healthcare personnel with SARS-CoV-2 infection (interim guidance). Centers for Disease Control and Prevention website. https://www.cdc.gov/coronavirus/2019-ncov/hcp/return-to-work. html. Published 2020. Accessed November 2, 2020. 